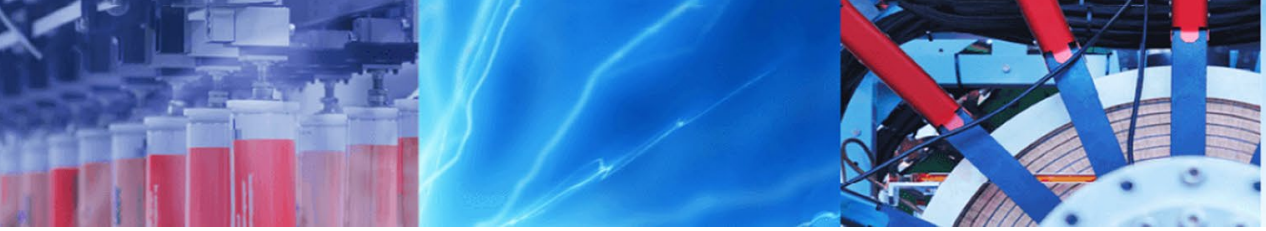

Research Article

\title{
Fault severity detection of a worm gearbox based on several feature extraction methods through a developed condition monitoring system
}

\author{
Berkan Hızarcı ${ }^{1}\left[\right.$ ] Rafet Can Ümütlü ${ }^{1} \cdot$ Zeki Kıral $^{2} \cdot$ Hasan Öztürk ${ }^{2}$
}

Received: 20 August 2020 / Accepted: 30 December 2020 / Published online: 12 January 2021

(C) The Author(s) 2021 OPEN

\begin{abstract}
This study presents the severity detection of pitting faults on worm gearbox through the assessment of fault features extracted from the gearbox vibration data. Fault severity assessment on worm gearbox is conducted by the developed condition monitoring instrument with observing not only traditional but also multidisciplinary features. It is well known that the sliding motion between the worm gear and wheel gear causes difficulties about fault detection on worm gearboxes. Therefore, continuous monitoring and observation of different types of fault features are very important, especially for worm gearboxes. Therefore, in this study, time-domain statistics, the features of evaluated vibration analysis method and Poincaré plot are examined for fault severity detection on worm gearbox. The most reliable features for fault detection on worm gearbox are determined via the parallel coordinate plot. The abnormality detection during worm gearbox operation with the developed system is performed successfully by means of a decision tree.
\end{abstract}

Keywords Condition monitoring · Fault severity detection · Poincaré plot · Parallel coordinates plot · Worm gearbox

\section{Introduction}

Machinery failures can cause severe interruption to work schedules, increasing outcomes, lower product quality and risk of health to machine operators. Predictive maintenance provides detection of the failures in an early stage, reduction of significant damage, increase on performance and life of machines. Many predictive maintenance techniques such as vibration analysis, wear debris analysis, acoustic emission, temperature analysis have been developed for fault detection on gearboxes [1, 2]. Among these techniques, vibration analysis is the most appropriate and common technique for detection of machinery faults. It is possible to determine a type of failures and severity of defects by measuring and analysing vibration of machines, because vibration signals carry the signature of the fault [3]. Up to date, many researchers have proposed various vibration analysis methods for fault detection on mechanical equipment, e. g. time domain analysis [4], frequency domain analysis [5], enveloped analysis [6], vibration visualization methods [7], time-frequency analysis methods [8] and neural networks [9].

Worm gearboxes are commonly used in various fields of industrial applications such as presses, rolling mills, conveyors, elevators, mining industry machines, escalators, helicopters, wind turbines, etc. They provide more output torque and low velocity with changing the direction of rotation. Worm gearboxes include two gear components called worm screw gear and wheel gear. The power transmission between worm and wheel gear are provided with sliding motion [10]. The sliding motion between gears causes non-stationary vibration signals [11]. While faults on other type of the gearboxes cause periodic impacts around the gear mesh frequencies, such

$\triangle$ Berkan Hızarcl, berkan.hizarci@deu.edu.tr| ${ }^{1}$ The Graduate School of Natural and Applied Sciences, Department of Mechatronics Engineering, Dokuz Eylül University, İzmir, Turkey. ${ }^{2}$ Department of Mechanical Engineering, Dokuz Eylül University, İzmir, Turkey. 
distinctive symptoms are not obvious for worm gearboxes [12]. Therefore, the fault detection for this type of gearbox is a challenging problem and thus, there are limited studies about fault detection on worm gearboxes. Elasha et al. studied pitting detection in escalator's worm gearboxes using vibration analysis [13]. They used statistical metrics, envelope analysis and spectral kurtosis for vibration analysis techniques. In their study, the distinctive harmonics of worm gear frequency were interpreted the presence of pitting damage. Peng and Kessissoglou diagnosed faults in a worm gearbox using combined the oil and vibration analysis [14]. They concluded that oil analysis is a useful method for the detection of gear wear, and the vibration analysis of worm gearbox is more appropriate for identifying bearing failures. Waqar and Demetgul worked on fault diagnosis of worm gears by using thermal analysis multilayer perceptron neural network [15]. In their study, the vibration signals, sound signals and infrared thermography of worm gear were examined for normal and faulty conditions under different speeds. It has been observed from these studies that not only traditional methods but also multidisciplinary methods must be applied for efficient and reliable fault detection on worm gearbox. For this purpose, in this study, the evaluated vibration analysis method (EVAM) and fault detection using Poincaré plot is applied. EVAM provides detection of abnormalities with the help of energy variations in specific bands of frequency spectrum [16]. Poincaré plot helps visualization of the correlation between two consecutive data points, and therefore distinction of chaos from randomness in data. Although Poincaré plot is mostly utilized in medical studies, it has been recently preferred for describing the mechanical dynamics of chaotic nature either in normal operation or in faulty conditions [17]. However, there is no practical application of this method for worm gearboxes in the literature.

By the $1990 \mathrm{~s}$, advances in electronic and sensor technologies enabled the development of portable instrumentation for predictive maintenance. Microcontrollers, embedded systems, field-programmable gate arrays (FPGA), digital signal processors (DSP), etc., are devoted to portable systems for condition monitoring (CM) field [18]. Nowadays, portable condition monitoring instruments are widely used in almost all industries for data acquisition and fault detection. They have some advantages such as low-cost, portability, simplicity, fast measurement, realtime monitoring, including powerful analysis methods, multichannel measurement, quick startup, reduction in system size and so on. To improve the performance of the portable CM systems up to date, several works have been studied. Zhang and Chen studied in condition monitoring of CNC milling machine tools [19]. Their aims are to enhance the quality, productivity of machined products and reduce the machining cost by detecting tool failures using three axes accelerometers and microcontroller. Betta et al. develop a diagnosis system for detecting faults of asynchronous AC motor using two concurrent DSP in real time [20]. Their study includes frequency spectrums of vibration signals and a pattern matching procedure. Camacho et al. develop FPGA based reconfigurable system in order to monitor cutting tool condition in highspeed machining process [21]. Son et al. [22] conducted an experimental study on the fault diagnosis of wind turbines through a condition monitoring system which is stated to be useful for the operation and maintenance.

Up to date, although fault detection on worm gearbox is investigated for many researchers, the subject is still an important area to be examined. The prior contribution of the research is not only analysing the conventional methods but also applying multidisciplinary methods such as Poincaré plot in the subject of fault detection for worm gearboxes. Furthermore, it must be noted that these feature extraction methods are applied in the developed portable real-time condition monitoring (CM) instrument unlike the other studies presented in the literature. Thus, fault detection on the worm gearbox is conducted in a reliable and fast way. In this study, time-domain statistical features, EVAM feature and the features of Poincaré plot are examined. The aforementioned methods have been chosen because of their real-time applicability in the designed $\mathrm{CM}$ device. After the analyses, the most reliable feature for fault detection on the worm gearbox is determined by the parallel coordinates plot. The fault detection by the developed system is realized with a decision tree with surveillance of the dominant feature. Processing steps of the proposed feature extraction methods can be shown in Fig. 1. It can be seen from the Figure. 1 that time synchronous averaging is applied to the acquired acceleration signals from the worm gearbox, then valuable features of time domain, EVAM and Poincaré plot are observed.

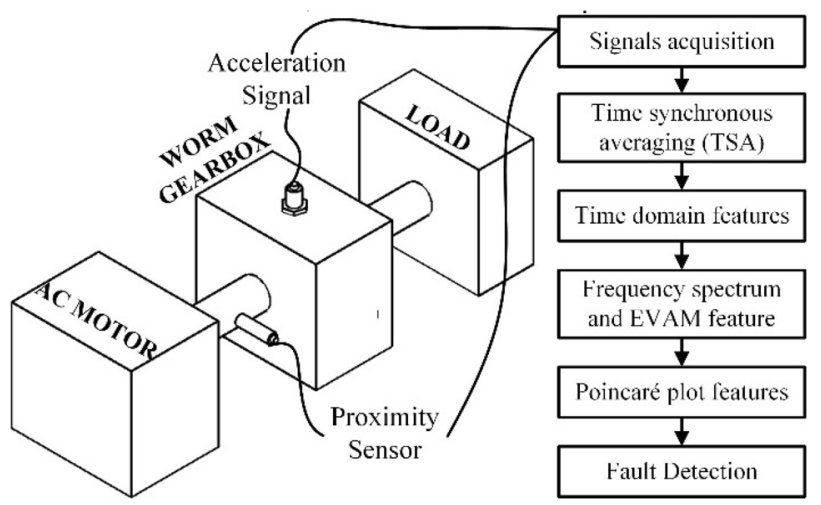

Fig. 1 Processing steps of the proposed feature extraction methods 
The paper is organized as follows: Section. 2 describes the theoretical background of pre-processing and feature extraction methods investigated in this study. Section 3 introduces experimental setup, and the different severity of pitting faults stimulated on wheel gear of worm gearbox are presented in Sect. 4. The analysis and results of the fault detection methods are given in Sect. 5. The determination of dominant features and fault severity detection on worm gearbox are conducted in Sect. 6 and Sect. 7, respectively. Finally, Sect. 8 presents the conclusions of this study.

\section{Theoretical background}

\subsection{Pre-processing}

Design of a vibration analyser represents a certain challenge. First of all, to be able to collect reliable data, operational conditions must be known and synchronously associated with the measured data. For example, rotating speed changes information in measured data. Without knowledge of rotating speed, interpretation of condition of a gearbox is not reliable. Second, vibration should be recorded without delay and almost in real time. Moreover, for dependable results, multiplexer scanning, which means taking a measurement of more than one, has to be done. The reliable analysis requires capable detection methods and continuous monitoring. Continuous monitoring generates vast amounts of data, thus these systems need computing powers and data storages. In this work, developed microcontroller based vibration analyser has been carefully designed regarding these aspects. For data storage, the USB drive is utilized. The all vibration analysis results are visualized on TFT screen to inform the operator. Moreover, the velocity of the worm gear is measured by a non-contact inductive proximity sensor. An accelerometer is mounted on the top of the worm gearbox which is the intersection area of worm and wheel gear since sensors should be placed in close vicinity to vibration sources in order to acquire good vibration response characteristics. Furthermore, time synchronous average (TSA) method is applied because gear faults symptoms occur synchronously with gear revolution. Distinctive symptoms may disappear because of the irrelevant noises. TSA removes this noise component from a vibration signal and extracts essential vibration signal which is related to gearbox. A simplified block diagram of TSA used in this study is shown in Fig. 2. It can be seen from the Fig. 2 that the vibration signal is averaged with the aid of trigger pulse from an inductive sensor measuring revolution of gear. The process continues until the desired number of averages $(\mathrm{N})$.

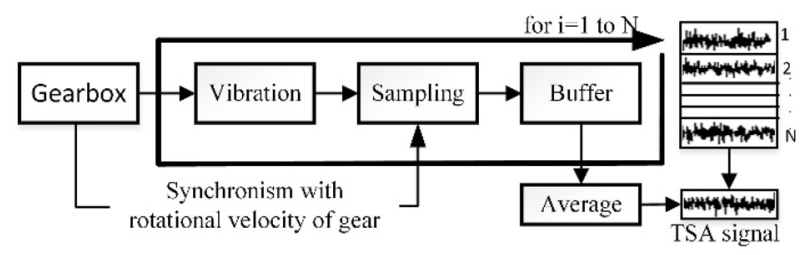

Fig. 2 A simplified block diagram of TSA

The TSA signal can be obtained using the Eq. (1), where $T$ denotes the period of gear rotation, and $\mathrm{N}$ is the number of averages. After performing a sufficient number of averages, vibrations which are related to the gear will remain, while non-related vibrations will be averaged and disappeared.

$X_{a v}(t)=\frac{1}{N} \sum_{k=1}^{N} x(t+k T)$

\subsection{Time-domain statistical features}

Time domain analysis of vibration signals is one of the traditional methods for interpreting rotating machine condition. This method examines the statistical properties of a vibration signal and enables detection of changes in the vibration signal caused by faults, but it is difficult to diagnose the source of changes. The most common statistical parameters in time domain are given in Table 1.

\subsection{Evaluated vibration analysis method}

Machinery failures and vibration frequencies have a close relationship. The faults within gearboxes show themselves in vibration signal at specific frequencies. Possible gearbox failures related to the vibration frequencies are given in Table 2 [23].

As seen in Table 2, the gear mesh frequency is a distinctive frequency for failures on gearboxes. The gear faults demonstrate itself as the increasing amplitude of gear mesh frequency and existence of its harmonics in the frequency spectrum. Gear mesh frequency (GMF) is the frequency at which gear teeth mate together in a gearbox. It is theoretically defined as the number of teeth on the gear multiplied by the rotational frequency of the gear. For the worm gearboxes, GMF is calculated as product of the number of thread on worm gear and worm gear frequency or product of the number of teeth on wheel gear and wheel gear frequency.

In order to illustrate frequencies in the vibration signal, time domain of the vibration signal is transformed into its frequency domain by using Fast Fourier 
Table 1 Statistical features

\begin{tabular}{|c|c|c|}
\hline Feature & Formula & Description \\
\hline Peak to peak value & $X_{\max }-X_{\min }$ & Determines difference between the highest to lowest amplitude value \\
\hline RMS value & $\sqrt{\frac{1}{N} \sum_{n=1}^{N}[x(n)]^{2}}$ & Determines effective magnitude of the signal \\
\hline Crest Factor Value & $\frac{\left|x_{\text {peak }}\right|}{x_{\text {rms }}}$ & Determines how extreme the peaks are in the waveform \\
\hline Kurtosis Value & $\frac{N \sum_{n=1}^{N}\left(x_{n}-\bar{x}\right)^{4}}{\left[\sum_{n=1}^{N}\left(x_{n}-\bar{x}\right)^{2}\right]^{2}}$ & Determines the heaviness of tail in signal \\
\hline Skewness Value & $\frac{N \sum_{i=1}^{N}\left(x_{n}-\bar{x}\right)^{3}}{\left[\sqrt{\frac{1}{N} \sum_{i=1}^{N}\left(x_{n}-\bar{x}\right)^{2}}\right]^{3}}$ & Determines the asymmetry of the signal about its mean value \\
\hline Mean Frequency Value & $\frac{1}{N} \sum_{k=1}^{N} \sum_{n=0}^{N-1} x_{n} e^{-i 2 \pi k n / N}$ & Determines mean value of a frequency spectrum \\
\hline
\end{tabular}

Table 2 Possible failures related to the vibration frequencies

\begin{tabular}{ll}
\hline Frequency & Possible failure \\
\hline High amplitudes at gear mesh frequency & Tooth error, Wear, Improper backlash, Overloaded gears \\
High energy levels at sidebands of gear mesh frequency & Gear tooth cracks, Eccentric gear, Non-parallel shafts \\
Existence of gear mesh frequency harmonics & Damaged gears, Incorrect tooth meshing, Tooth break-out \\
\hline
\end{tabular}

Transform (FFT) algorithm. The vibration signal and its FFT, denoted by $x(n)$ and $X(k)$, respectively, are expressed in Eq. (2), where $N$ is the number of points, $n$ is discrete time index, $k$ is frequency index and $k=0,1,2 \ldots N-1$.

$X(k)=\sum_{n=0}^{N-1} x(n) e^{-\frac{i 2 \pi}{N} k n}$

As mentioned before in Sect. 1, the increase around GMF is not obvious for worm gearbox in case of faults. Therefore, evaluated vibration analysis method which combines time domain analysis and frequency domain analysis has been developed [24]. This method can detect abnormalities in the amplitude of not only GMF but also its harmonics. By this method, the distinctive gear frequencies, its sidebands and harmonics are examined in frequency bands. It is well known the amplitude of distinctive frequencies changes in the frequency spectrum in case of faults. Evaluated vibration analysis method aims to detect these abnormal variations in the amplitude of these distinctive gear frequencies. For this purpose, the method calculates total RMS values of these frequencies in a bandwidth. Firstly, distinctive gear frequencies are determined. Band pass filters are applied on frequency spectrum so that the only these distinctive gear frequencies and sidebands passes. After, EVAM value is computed by using total RMS value of the bands, as given in Eq. (3) where B1, B2... BN stand for the considered frequency bands. The implementation of this method is illustrated in Fig. 3.

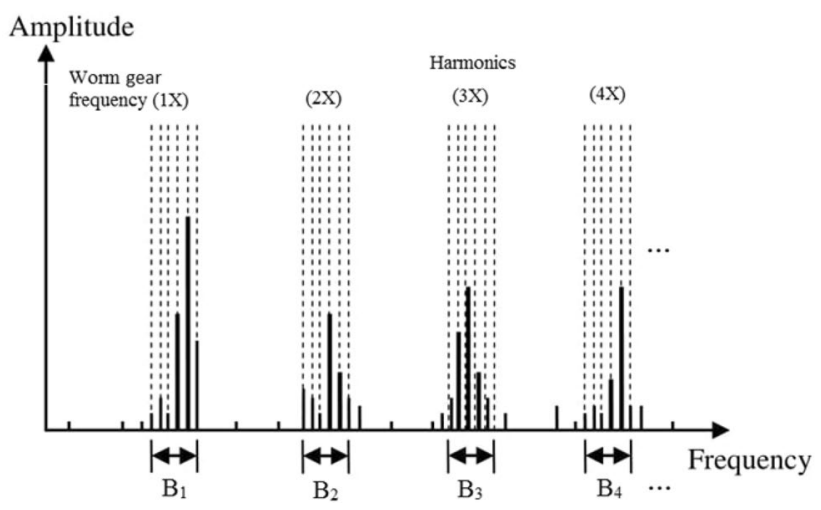

Fig. 3 The implementation of EVAM for worm gearbox

$E V A M=\sqrt{R M S_{B_{1}}{ }^{2}+R M S_{B_{2}}{ }^{2}+\ldots+R M S_{B_{N}}{ }^{2}}$

\subsection{Fault detection using Poincaré plot}

In this section, a method based on Poincaré plot is presented for a more reliable assessment of the condition of gearboxes. This method provides graphical examination of the vibration of the gearbox. Poincare plot is a type of recurrence plot which is a projection of the data set in phase space where every degree of freedom or parameter of the system is represented as an axis of a 
multidimensional space. Poincaré plots provide a way to visualize periodicities but also irregular cyclicities in natural processes. They are generally utilized to distinguish chaos from randomness in data set. In terms of fault detection on worm gearbox, in case of gear fault, the accelerations of gearbox changes and deviate from the normal operating condition. In such a case, the Poincaré plot of the gearbox shows abnormal behaviour and will be out of natural appearance. Poincare plots of the vibration signals are obtained by plotting each sample against the subsequent sample. The plot is formed on the screen by making the pixel value as " 0 " in the pixel corresponding to the acceleration values. This forms a black dot on the white "1" background. One black dot in the phase plane represents the acceleration point located thanks to two subsequent acceleration values of the gearbox. After all acceleration values are processed; the Poincaré plot of vibration data is appeared, as shown in the right side of Fig. 4.

The confidence ellipse of Poincaré plot and its semiminor and semi-major axis length can be seen in Fig. 4 . The confidence ellipse defines a region that contains $99 \%$ of all samples. For this study, this region gives an acceleration map of the gearbox during its operating time. The changes on the features of the region during operation give valuable information about condition of the gearbox. The features are area, semi-minor axis length and semi-major axis length of confidence ellipse of Poincaré plot.

For plotting confidence ellipse, a formula of center orient ellipse in the standard and matrix forms are given in Eq. (4) where $x$ and $y$ are the coordinates of any point on the ellipse, $a$ and $b$ are the radius on the $x$ and $y$ axes, respectively. Assuming $a>b$, $a$ is a semi-major axis length and $b$ is a semi-minor axis length [25]. The center orient ellipse may be rotated to a different orientation by a $2 \times 2$ rotation matrix which is given in Eq. (5) where $\varphi$ is the orientation angle.

$1=\frac{x^{2}}{a^{2}}+\frac{y^{2}}{b^{2}}=\left[\begin{array}{ll}x & y\end{array}\right]\left[\begin{array}{cc}\frac{1}{a^{2}} & 0 \\ 0 & \frac{1}{b^{2}}\end{array}\right]\left[\begin{array}{l}x \\ y\end{array}\right]=X^{T} D X$

$R=\left[\begin{array}{cc}\cos \phi & -\sin \phi \\ \sin \phi & \cos \phi\end{array}\right]$

The general transformation can be written $Y=R X$ with inverse $X=R^{-1} Y$, where $Y=[\bar{x} \bar{y}]^{T}$ is a new coordinates matrix of a rotated ellipse. The rotation matrix is orthogonal, therefore $R^{-1}=R^{T}$. Substituting this into Eq. (4) leads to,

$Y^{T} R D R^{T} Y=1$

After orientation, the ellipse can be translated so that its old center, the origin 0 , is mapped to a new center $K=\left[\begin{array}{ll}x_{0} & y_{0}\end{array}\right]^{T}$. The general transformation becomes $Y=K+R X$. Equation (6) is modified to include the translation,

$(Y-K)^{T} R D R^{T}(Y-K)=1$

Equation (7) is the ellipse equation after rotation and translation. The center (K), semi-major (a) and semi-minor of axis length (b) and orientation angle $(\varphi)$ must be known to form an ellipse. These parameters must be determined according to the data. For this purpose, the covariance matrix of the two dimensional data is calculated. The covariance matrix (C) defines the spread (variance) and the orientation (covariance) of the data. The largest eigenvector of the covariance matrix indicates the direction of the largest variance of the data, and the corresponding eigenvalue gives the largest spread in the direction of the eigenvector. This is also valid for the smallest eigenvector
Fig. 4 The process of Poincaré plot
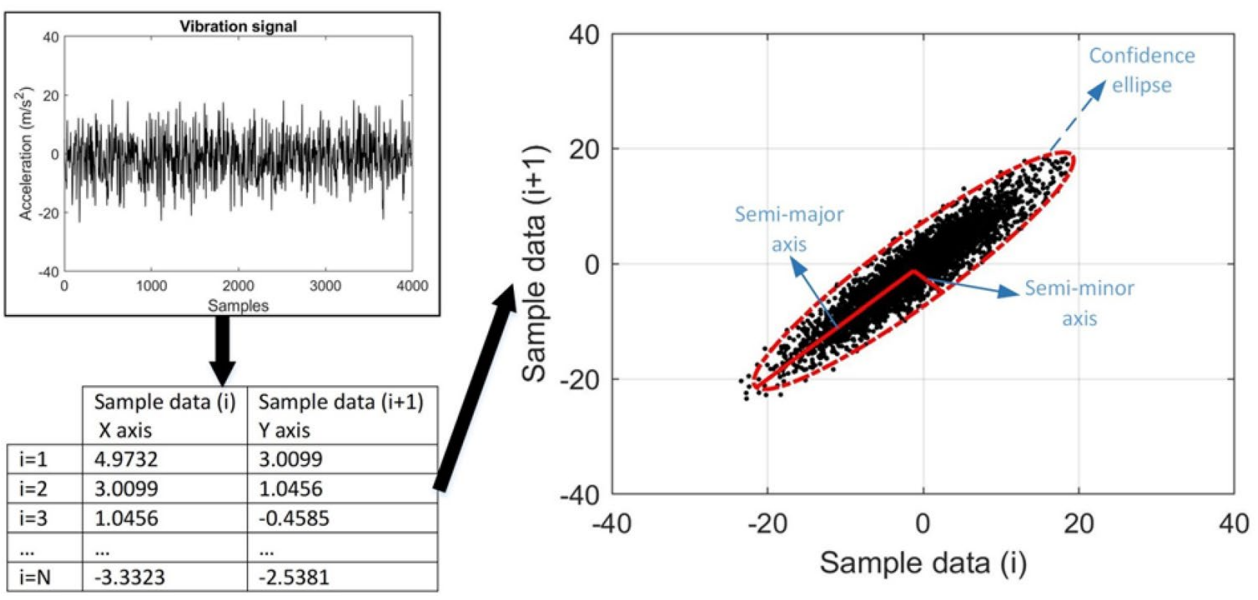
and eigenvalue. Therefore, the center (K), semi-major (a) and semi-minor of axis length (b) and orientation angle $(\varphi)$ of the ellipse can be determined by using eigenvalues and eigenvectors of the covariance matrix of the data. The covariance matrix is calculated for two dimensional data as given in Eq. (8) where $\sigma_{11}$ and $\sigma_{22}$ are variance of the data in $x$ direction and y direction, respectively. $\sigma_{12}$ is covariance between data in $x$ and $y$ direction, and $\sigma_{12}=\sigma_{21}$. The center (K), semi-major (a) and semi-minor of axis length (b) and orientation angle $(\varphi)$ of confidence ellipse are determined as given in Eq. (9).

$C=\left[\begin{array}{ll}\sigma_{11} & \sigma_{12} \\ \sigma_{21} & \sigma_{22}\end{array}\right]$

$K=\left[\begin{array}{ll}\mu_{x} & \mu_{y}\end{array}\right] a=\sqrt{s \lambda_{1}} b=\sqrt{s \lambda_{2}}$

$\phi=\frac{1}{2} \arctan \frac{2 \sigma_{12}}{\sigma_{22}-\sigma_{11}}$

$\lambda_{1,2}=\frac{1}{2}\left(\sigma_{11}+\sigma_{22} \pm \sqrt{\left(\sigma_{11}-\sigma_{22}\right)^{2}+4 \sigma_{12}^{2}}\right)$

$\mu_{\mathrm{x}}$ and $\mu_{\mathrm{y}}$ are mean values of the data in $\mathrm{x}$ and $\mathrm{y}$ direction, $\lambda_{1}$ and $\lambda_{2}$ are largest and smallest eigenvalues of the covariance matrix and $s$ is a scaling factor of the ellipse which is related to the confidence level of the data. The data acquired from the acceleration sensor is normally distributed. Therefore, the left hand side of Eq. (4) actually represents the sum of squares of independent normally distributed data samples.

The sum of squared Gaussian data becomes Chi-Square distribution. For a Chi-Square distribution, 99\% confidence level with two degree of freedom corresponds to $s=9.210$ [26]. Figure 5 shows the confidence ellipses of the data for several confidence levels. The confidence level is chosen as $99 \%$ because all black dots on the Poincaré plot are important to understand the behaviour of the gearbox.

\section{Experimental setup}

The experimental system consists of an AC motor $(2.2 \mathrm{~kW}$, $3000 \mathrm{rpm}, 3$ phase), a worm gearbox (1:15 gear ratio) and an electromagnetic brake ( $40 \mathrm{Nm}$ maximum braking torque). It consists of 2 thread worm gear and 30 teeth wheel gear. The operation conditions of experiments are shown in Table 3.

The experimental test rig is illustrated in Fig. 6. The AC motor is connected to the worm gearbox via two v-belts (1:1 ratio) in order to avoid transferring motor vibrations to the gearbox. The worm gearbox's output shaft and electromechanical brake are connected via a torsionally flexible coupling.

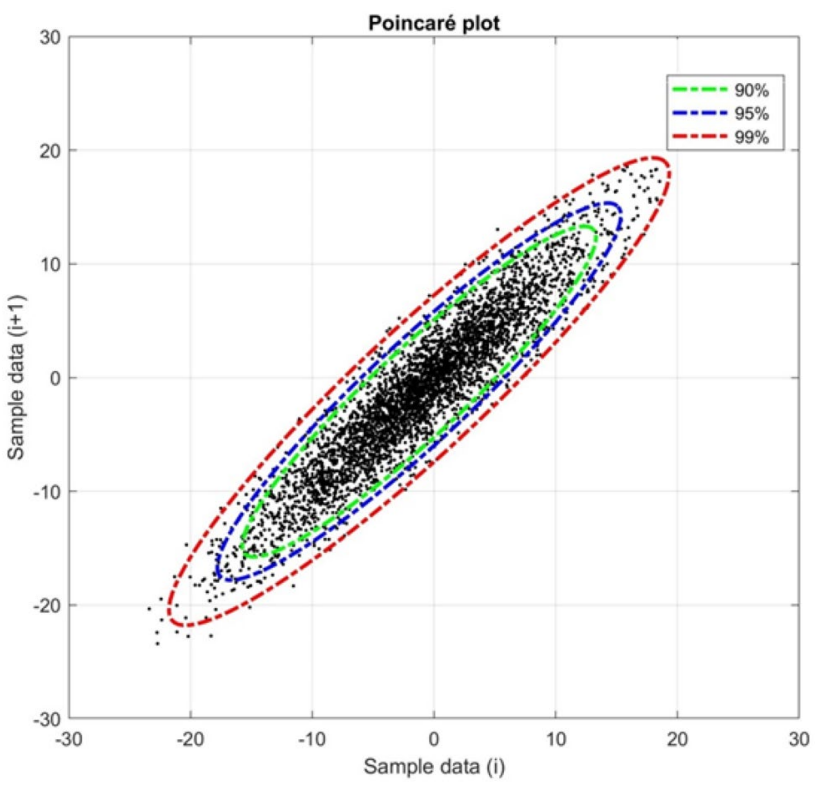

Fig. 5 The confidence ellipses of the data for several confidence levels

Table 3 Operation conditionsf

\begin{tabular}{ll}
\hline Angular velocity of the worm gearbox input shaft & $3012 \mathrm{rpm}$ \\
\hline Angular velocity of the worm gearbox output shaft & $200.8 \mathrm{rpm}$ \\
Output torque of the worm gearbox & $84.7 \mathrm{Nm}$ \\
Brake torque & $38.4 \mathrm{Nm}$ \\
Worm gear frequency & $50.2 \mathrm{~Hz}$ \\
Gear mesh frequency (GMF) & $100.4 \mathrm{~Hz}$
\end{tabular}

In this study, PCB Piezotronics 352A76 series high-resolution piezoelectric accelerometers are used to measure acceleration of gearbox. PCB Piezotronics $480 \mathrm{C0} 2$ series signal conditioner is utilized for conditioning output signals, voltage follower, AC or DC coupling. Vibration analyser and abnormality detection instrument is utilized for data acquisition, analyser, visualization and decision module. For this purpose, STM32F429 from STMicroelectronics is preferred since it includes highperformance ARM Cortex M4 core microcontroller which is very powerful for data acquisition and digital signal processing instructions in real-time. Throughout the study, the sampling frequency is chosen as $2 \mathrm{kHz}$ according to the Nyquist theorem, since the important frequencies of this study are below $1 \mathrm{kHz}$. The number of data points used for computing the frequency spectrum $(\mathrm{N})$ equals 2048. After the vibration signal analysis is completed, results of the analysis are displayed on the TFT screen and vibration data are stored on USB flash drive. 
Fig. 6 The experimental test rig

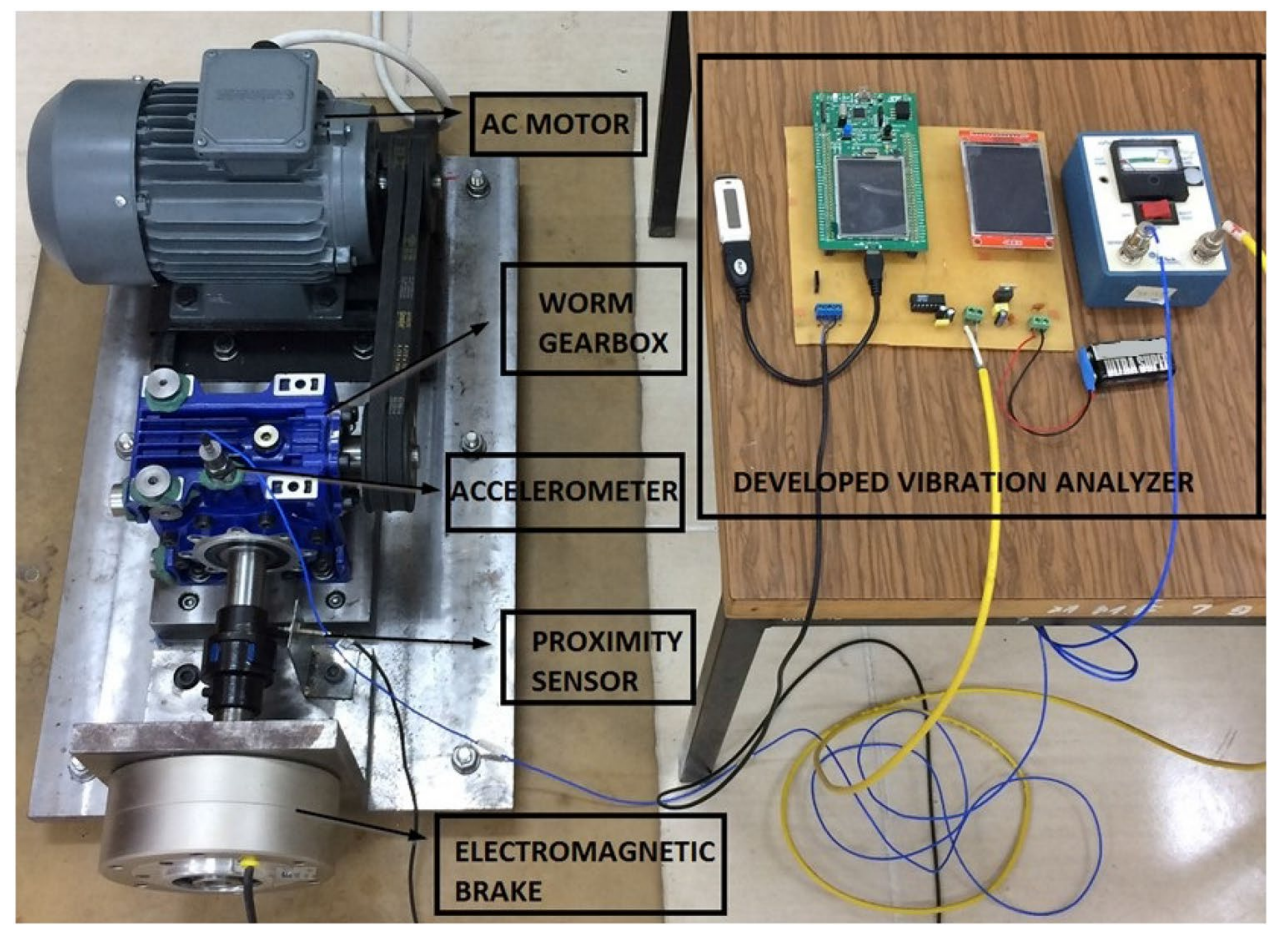

\section{Simulation pitting faults}

Destructive pitting faults may occur in time on the wheel tooth surface on worm gearboxes because of the higher load than the capacity of the gear. Pitting failure is also characterized according to the diameter of pits, such as initial pitting and destructive pitting. Initial pits are generally from 0.4 to $1 \mathrm{~mm}$ in diameter. Destructive pitting is considerably larger in diameter than those associated with initial pitting. The pitting faults also can be local or distributed on gear surface. In this study, destructive distributed elliptical pitting faults whose diameter and depth are approximately $2 \mathrm{~mm}$ and $1 \mathrm{~mm}$ respectively have been implemented artificially on wheel gear. First, five artificial local surface pits were introduced on one of the wheel gear teeth for Fault 1, as shown in Fig. 7a. For simulation of spreads of faults, artificial pits are introduced over the neighbouring teeth surfaces as shown Fault 2 in Fig. 7b. The number of simulated pits was increased progressively in order to represent the development of the pitting fault as illustrated Fault 3 in Fig. 7c.

\section{Analysis and results}

\subsection{Statistical features}

In this section, time domain parameters of worm gearbox are examined for the cases of healthy and faulty conditions of the equipment. These statistical parameters are calculated from the TSA vibration signal. The results of the time domain analysis are given in Fig. 8.
Fig. 7 The artificial pitting faults on the wheel gear

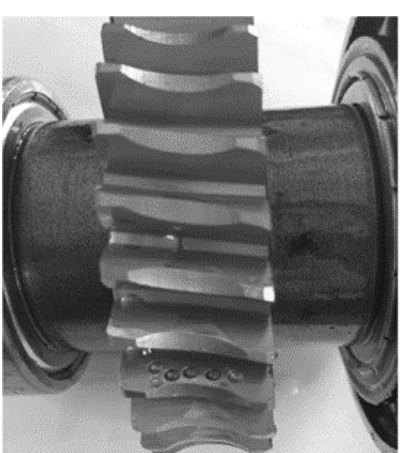

(a) Fault 1

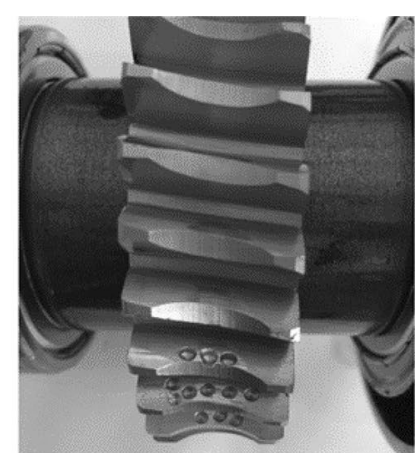

(b) Fault 2

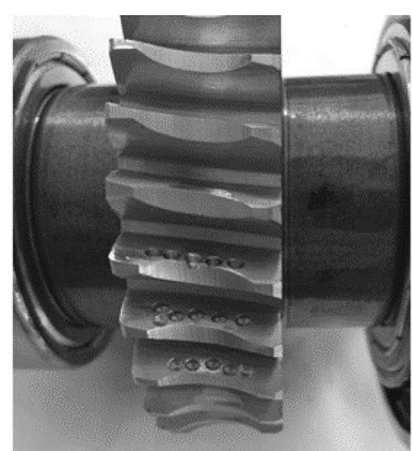

(c) Fault 3 

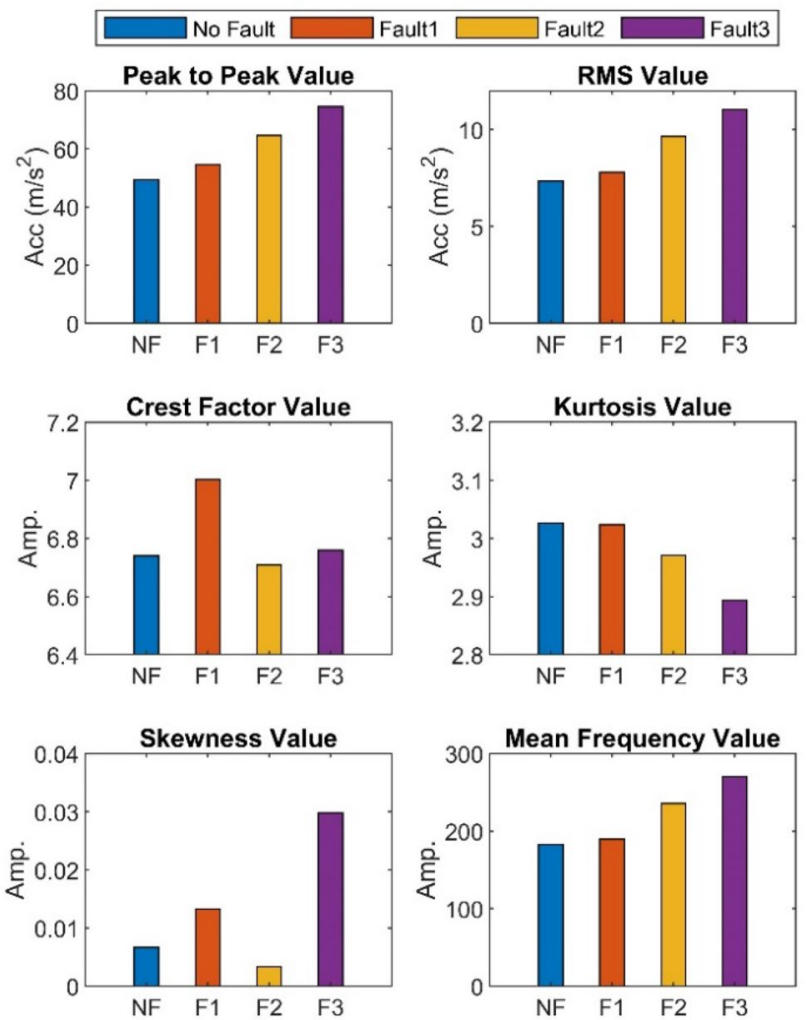

Fig. 8 The changes on time-domain statistical features

As shown in Fig. 8, RMS, peak to peak and mean frequency values exhibit an increasing trend with the fault progression, although Crest Factor and Skewness values do not show the fault progression. Increasing the magnitude of the faults causes the value of kurtosis to decrease. Peak to peak value shows increasing trend with the fault progress because higher positive and negative peaks in vibration signal occur as the severity of pitting faults increases. The increasing faults affect also RMS value of vibration signal progressively because the power of the vibration signal rises. Moreover, mean of the amplitude of frequency domain increases with the fault progression. This means; the amplitude of frequency spectrum increases as the severity of fault increases.

\subsection{Evaluated vibration analysis method}

In this method, time domain vibration signal measured on worm gearbox are transformed into frequency domain. Frequency spectrums of vibration signals are given in Fig. 9.

According to Fig. 9, although the gear mesh frequency (GMF) is not observed in the frequency spectrum for No Fault condition, it is in the frequency spectrums of Fault 1,2 and 3. Thus, it can be stated that GMF appears in the

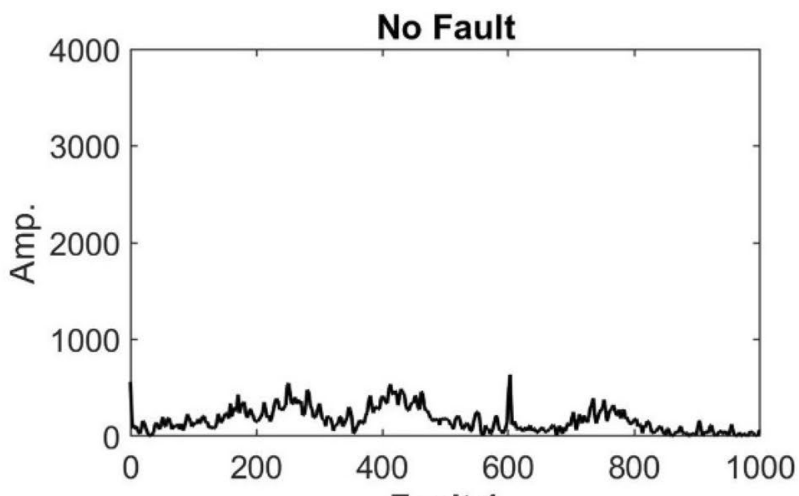

Fault 1

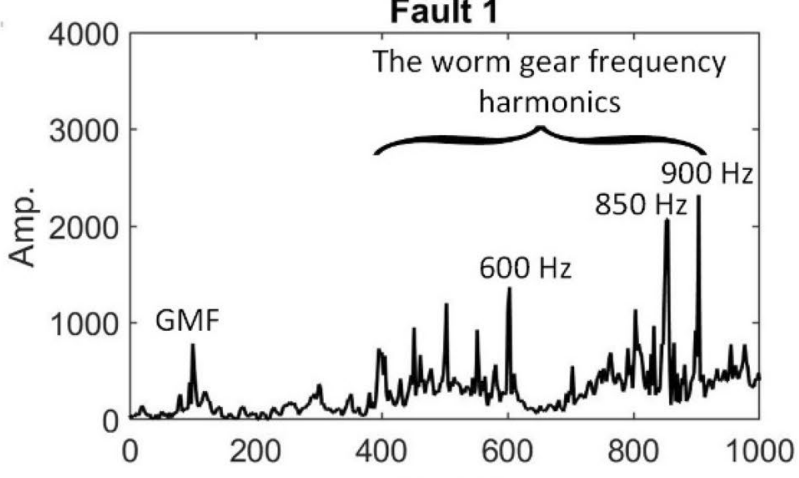

Fault 2

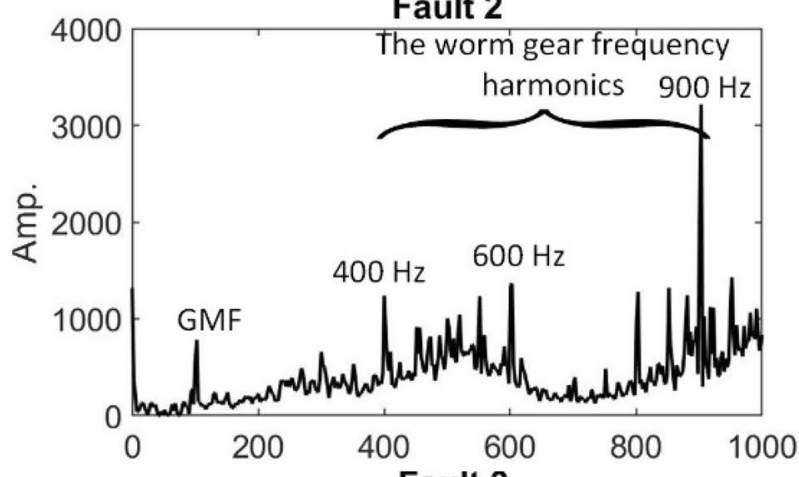

Fault 3

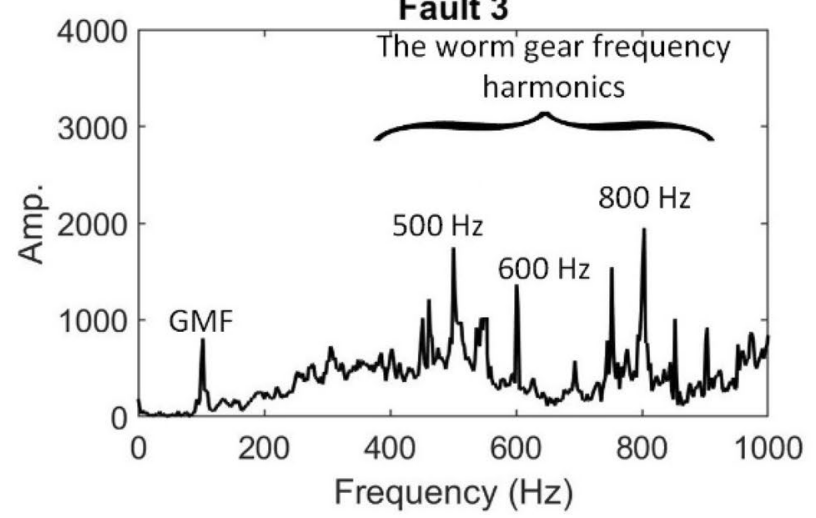

Fig. 9 Frequency spectrums of vibration signals for each condition

frequency spectrum in case of faults on worm gearbox. Moreover, the amplitude of spectrums increases especially at the high frequencies with the faults progression. It is seen that the peaks become visible at the worm gear 
frequency harmonics. As a result, the existence of GMF and harmonics of worm gear frequency are interpreted as the pitting failure effects on the frequency spectrum of worm gearbox.

For EVAM method, the eighteen band pass filters (BPFs) whose center bandwidths are between $45-50 \mathrm{~Hz}$, 95-105 Hz ... 895-905 Hz are designed. After these BPFs apply to the frequency spectrum of the vibration signal, the amplitudes of frequencies inside the bandwidths are

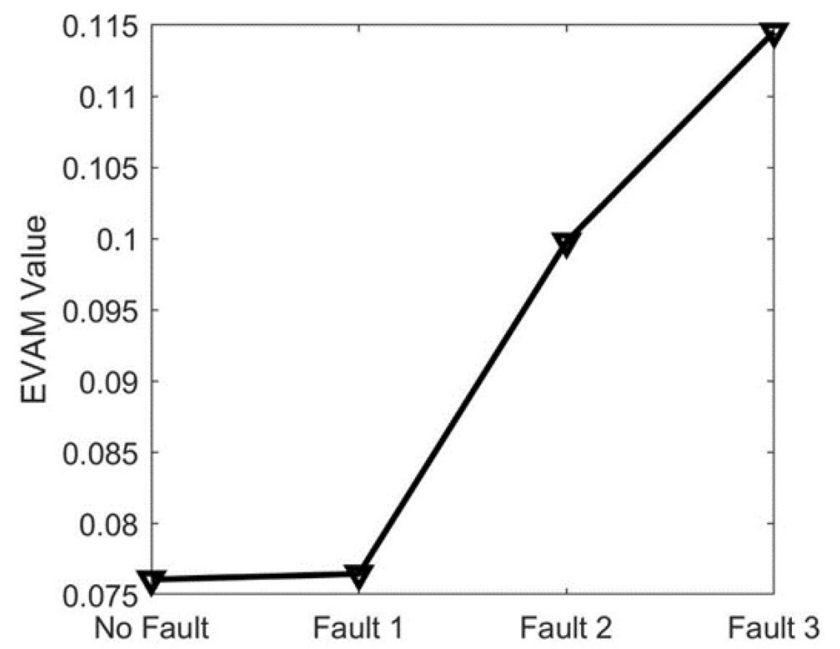

only passed, the others are rejected. The EVAM value is calculated for these frequencies according to Eq. (3). Results of EVAM for each condition are given in Fig. 10. It can be seen from Fig. 10 that the EVAM values for No Fault and Fault 1 conditions are very close. Yet, as the severity (or number) of pits is increased, EVAM exhibits fault symptoms as an increase. This means; the amplitude of worm gear frequency and its harmonics increase with the fault progression after the Fault 1.

\subsection{Fault detection using poincaré plot}

Performance of this method has been investigated for each condition of the gearbox. In this method, the TSA vibration signals have been utilized for simplicity in the visualization of results. The results are shown in Fig. 11. Each data contains 3983 samples. The center, area, semiminor and semi-major axis lengths of confidence ellipses are used for the evaluation process. The area of the confidence ellipse (A) is calculated as $A=\pi \times a \times b$.

As can be seen from Fig. 11, it is obvious that the area of confidence ellipse enlarges progressively from No Fault to Fault 3 condition. For No Fault condition, black dots are often clustered in the center. On the other hand, it has been observed that the black dots spread out from the center as the fault progress. The averaged values of features for each condition can be seen in Table 4 .

Fig. 10 The results of EVAM

Fig. 11 Poincaré plots for all conditions
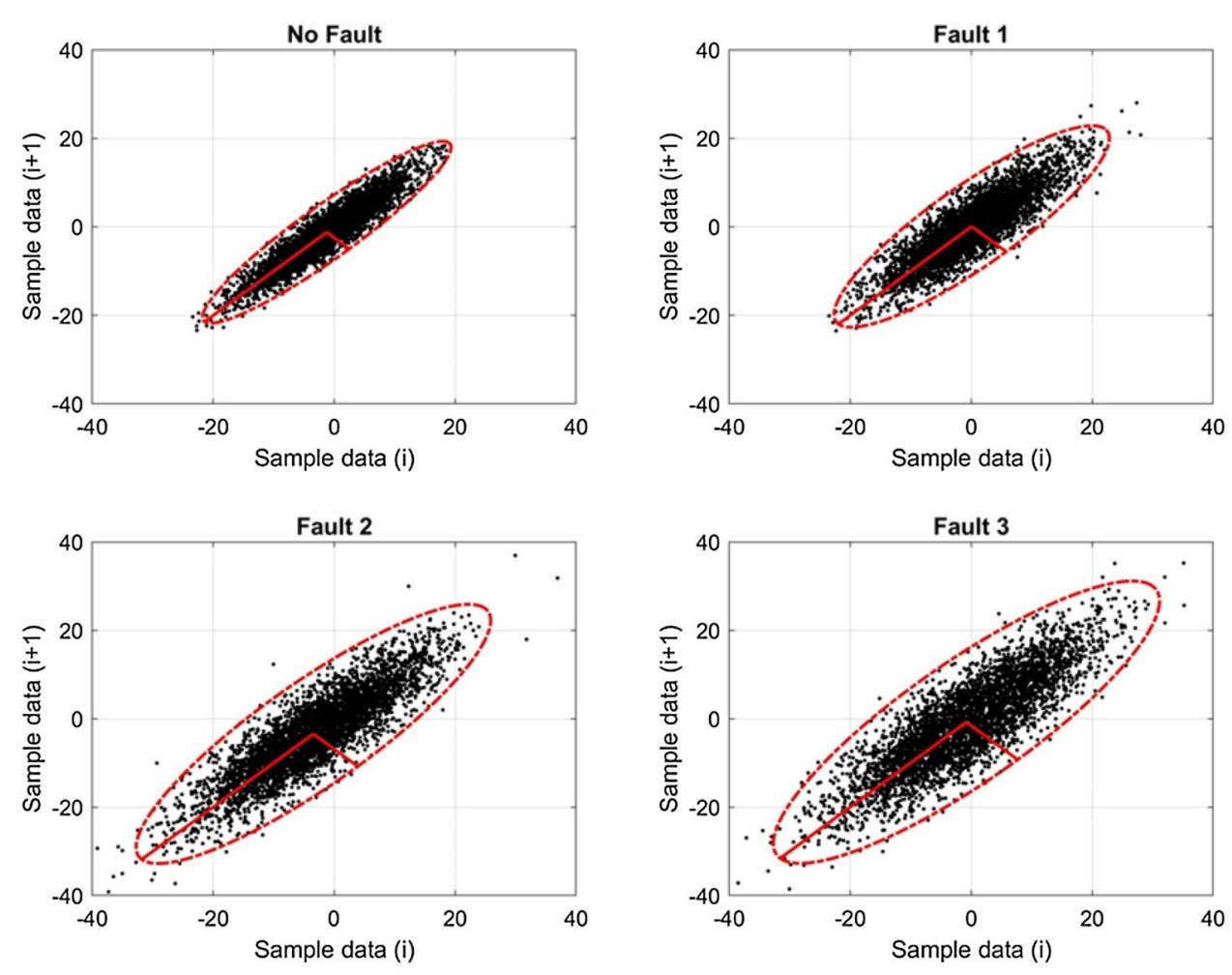
Table 4 The Poincaré features

\begin{tabular}{llll}
\hline & $\begin{array}{l}\text { Semi-major axis } \\
\text { length }(\mathrm{a})\end{array}$ & $\begin{array}{l}\text { Semi-minor axis } \\
\text { length }(\mathrm{b})\end{array}$ & Area (A) \\
\hline No Fault & 28.803 & 5.346 & 483.777 \\
Fault 1 & 30.973 & 8.087 & 786.894 \\
Fault 2 & 40.142 & 10.418 & 1313.8 \\
Fault 3 & 43.551 & 12.086 & 1653.6 \\
\hline
\end{tabular}

Table 4 shows that the area of the confidence ellipse increase $63 \%$ between No Fault and Fault 1, 67\% between Fault 1 and Fault 2, 26\% between Fault 2 and Fault 3 cases. Semi-major and semi-minor axis lengths increase with the advancement of the fault severity. According to the results, these features give useful information about the severity of faults and condition of the gearbox.

\section{Feature selection}

The fault detection methods used in this study have some features that make it possible to notice the abnormality of the worm gearbox. The results presented in Sect. 5 show that these distinctive features are RMS and mean frequency value from time domain analysis, value from EVAM, area, semi-major and semi-minor axis length from Poincaré plot. Although visual inspection of these features is sufficient to detect the faults, there is a need for a faster and reliable automated abnormality detection procedure. For detection abnormalities, the prediction values of these features at normal operation of the gearbox are compared with the measured values, and an alarm signal is triggered when a significant gap between values appears. It is known that the features providing more significant and reliable gap are more imperious or distinctive in terms of fault detection. Performing the analysis of these dominant features first increases the accuracy of abnormality detection. For this purpose, the parallel coordinates plot is applied to decide dominant features that have high variance or different distributions. The parallel coordinates plot is the type of visualization of multivariate numerical data to compare variables and see the relationship between them. The aforementioned distinctive features for this study are each represented with a vertical line for 800 condition feature values in Fig. 12. The feature values are calculated for each revolution of the worm gearbox and there are 800 samples for a total of 4 conditions and 200 revolutions.

It can be seen from Fig. 12 that EVAM value is the worst feature to detect abnormalities caused by faults as it cannot be distinguished due to the crossing lines. Although RMS, Mean frequency and semi-major axis length values for Fault 2 and Fault 3 can be separated, these features intertwine for No Fault and Fault 1. However, it is observed that area and semi minor axis length of the Poincare plot is the most distinguishable features to determine conditions of the worm gearbox. Therefore, area and semi minor axis length of the Poincaré plot are utilized to decide condition of the gearbox and detect abnormalities.

\section{Fault severity detection}

This part of the study focuses on determination of abnormality situations with the use of decision tree. Decision tree is one of the information approaches carried out to classify feature vectors based on their success in abnormality detection. The feature that presents at the root node of the decision tree is regarded as the most reliable feature for abnormality detection. The remaining features which could contribute the detection appear at the successive nodes in descending order of detection success. However, the features that do not have any contribution are discarded from the tree. This concept provides selecting successful features in terms of abnormality detection, thus more reliable and faster detection can be conducted. With the aid of decision tree, the abnormality detection and alarm management is conducted in this study for developed CM system. The features of area and semi minor axis length of the Poincaré plot are supplied as inputs to the decision tree. The trained decision tree with a c4.5 algorithm which is the most common algorithm to construct decision trees is as shown in Fig. 13.

The decision tree in Fig. 13 shows that area of the Poincaré plot is sufficient feature to determine the condition of the worm gearbox. Therefore, the semi minor axis length is discarded from the decision tree as a result of the $c 4.5$ algorithm. The values of the area of Poincare plot on the decision tree in Fig. 13 are provided by the algorithm after the training process. The values of the area of Poincaré plot feature in Fig. 13 have emerged considering the maximum-minimum characteristic of all trained data for all conditions. For instance, the values up to 635.335 are accepted as No fault condition since the minimum value of area of Poincaré plot in all Fault 1 data is found as 635.335 by the decision tree algorithm. When the value of this feature is greater than 635.335 , it is considered that the abnormality starts and Fault 1 occurs. Fault 2 occurs for values greater than 1050.35, and Fault 3 occurs for values greater than 1483.69. The reason for the difference between the values in Fig. 13 and Table 4 is that the values given in Table 4 are average. Finally, the alarm management according to the classification of the fault conditions is conducted with the intervals of Poincaré area values as given in Fig. 13. 


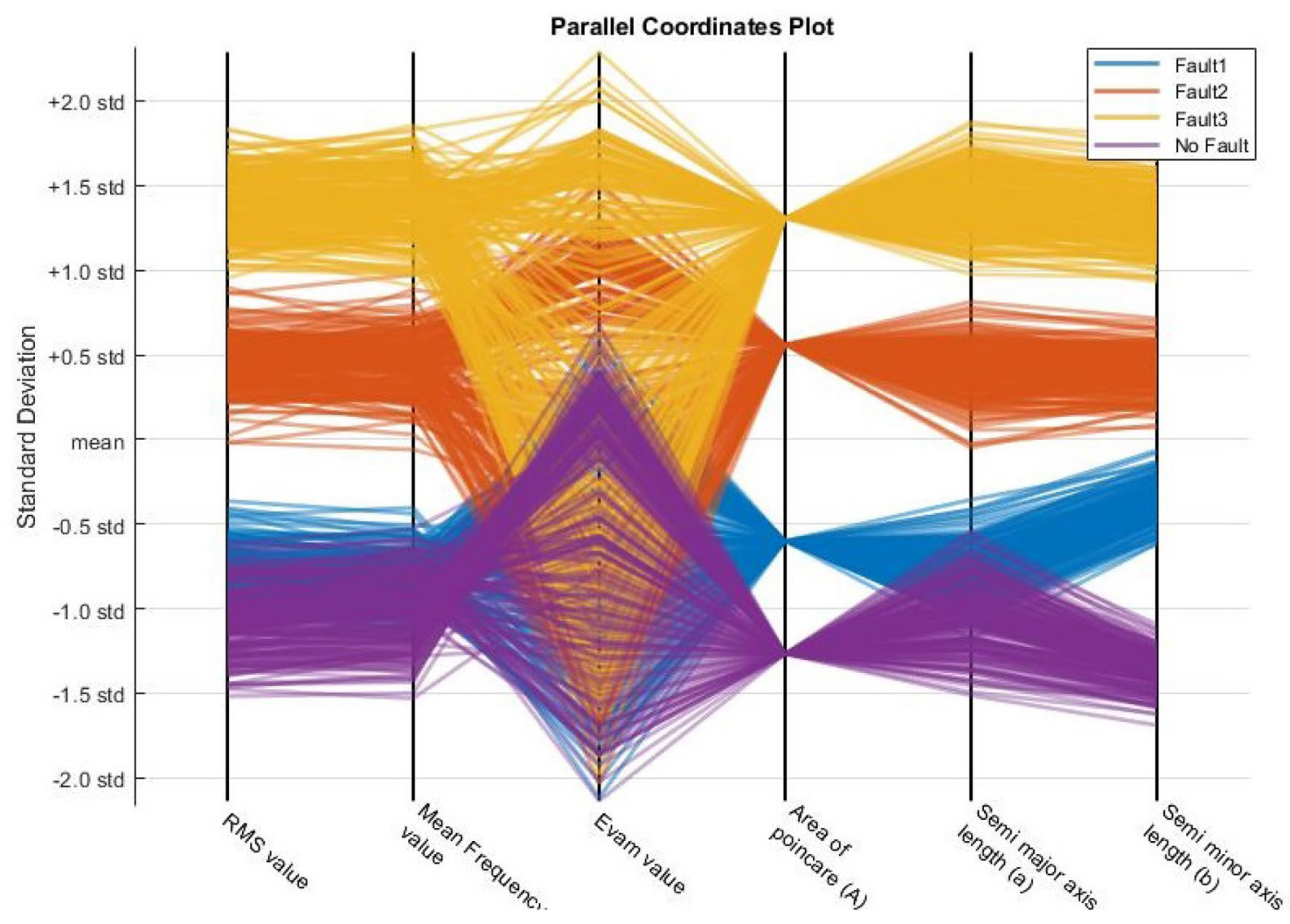

Fig. 12 The decision tree of the dominant features

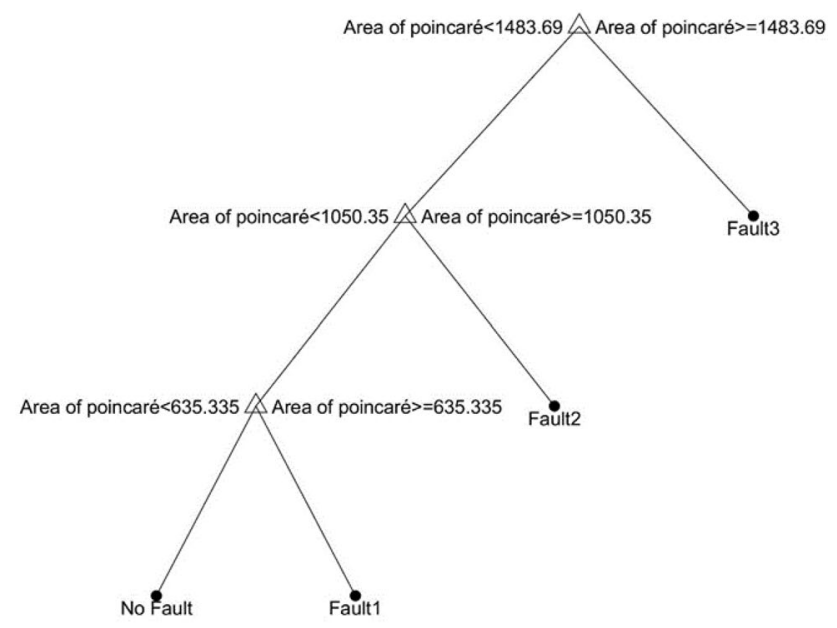

Fig. 13 The decision tree for the dominant features

\section{Conclusion}

In this study, a microcontroller-based condition monitoring and abnormality detection system is developed. To test the developed instrument, the experiments were conducted under increasing severity of simulated pitting faults on worm gearbox. For detection of the faults, traditional and multidisciplinary methods were applied. According to time-domain statistical features, it is seen that the increasing severity of faults affects the RMS and mean frequency value of vibration signal progressively. In the evaluated vibration analysis method, EVAM values show an increase especially for Fault 2 and 3 due to high amplitude at the worm gear frequency and harmonics in the frequency spectrum. Fault detection using Poincaré plot method detects the pitting faults thanks to its valuable features. The distinctive features from all methods are visualized with parallel coordinates plot to get most dominant features for fault detection on worm gearbox. To get more reliable, fast and automatic abnormality decision with the developed system, the decision tree is applied. Consequently, the results show that the area of Poincaré plot is the most dominant feature to determine the condition of worm gearbox and abnormality detection. The fault severity of worm gearbox can be assessed with this feature automatically by a microcontroller-based condition monitoring system. It is planned that this study will be extended for fault detection of other mechanical equipment such as other gearbox types and bearings so that a single condition monitoring system will detect faults for all types of rotating machine components. 
Acknowledgements This research did not receive any specific grant from funding agencies in the public, commercial, or not-for-profit sectors.

\section{Compliance with ethical standards}

Conflict of interest The authors declare that they have no conflict of interest.

Open Access This article is licensed under a Creative Commons Attribution 4.0 International License, which permits use, sharing, adaptation, distribution and reproduction in any medium or format, as long as you give appropriate credit to the original author(s) and the source, provide a link to the Creative Commons licence, and indicate if changes were made. The images or other third party material in this article are included in the article's Creative Commons licence, unless indicated otherwise in a credit line to the material. If material is not included in the article's Creative Commons licence and your intended use is not permitted by statutory regulation or exceeds the permitted use, you will need to obtain permission directly from the copyright holder. To view a copy of this licence, visit http://creativecommons. org/licenses/by/4.0/.

\section{References}

1. Öztürk H, Sabuncu M, Yesilyurt I (2008) Early detection of pitting damage in gears using mean frequency of scalogram. J Vib Control 14:469-484

2. Amarnath M, Lee SK (2015) Assessment of surface contact fatigue failure in a spur geared system based on the tribological and vibration parameter analysis. Measurement 76:32-44

3. Marquez FPG, Tobias AM, Perez JMP, Papaelias M (2012) Condition monitoring of wind turbines: techniques and methods. Renewable Energy 46:169-178

4. Yunusa-Kaltungo A, Sinha JK, Elbhbah K (2014) An improved data fusion technique for faults diagnosis in rotating machines. Measurement 58:27-32

5. Zhou L, Duan F, Mba D, Wang W, Ojolo S (2018) Using frequency domain analysis techniques for diagnosis of planetary bearing defect in a CH-46E helicopter aft gearbox. Eng Fail Anal 92:71-83

6. Yizhou Y, Yang W, Jiang D (2018) Simulation and experimental analysis of rolling element bearing fault in rotor-bearing-casing system. Eng. Fail. Anal. 92:205-221

7. B. Hizarci, R. C. Ümütlü, H. Ozturk and Z. Kıral, Vibration region analysis for condition monitoring of gearboxes using image processing and neural networks, Exp. Tech., (2019) 1-17.

8. Ramteke DS, Parey A, Pachori RB (2019) Automated gear fault detection of micron level wear in bevel gears using variational mode decomposition. J Mech Sci Technol 33(12):5769-5777

9. Jami A, Heyns PS (2018) Impeller fault detection under variable flow conditions based on three feature extraction methods and artificial neural networks. J Mech Sci Technol 32(9):4079-4087

10. A. Black, The ins and outs of worm gears, http://www.machineryl ubrication.com/Read/1080/worm-gears, accessed 24 July 2020.
11. Dudas I (2000) The theory and practice of worm gear drives. Penton Press, London

12. P. Vahaoja, S. Lahdelma and J. Leinonen, (2006) On the condition monitoring of worm gears, 1st World Congress on Engineering Asset Management Conference, 332-343.

13. Elasha F, Carcel CR, Mba D, Kiat G, Nze I, Yebra G (2014) Pitting detection in worm gearboxes with vibration analysis. Eng Fail Anal 42:366-376

14. Peng Z, Kessissoglou NJ (2003) An integrated approach to fault diagnosis of machinery using wear debris and vibration analysis. Wear 255:1221-1232

15. Waqar T, Demetgul M (2016) Thermal analysis MLP neural network based fault diagnosis on worm gears. Measurement 86:56-66

16. Zamanian AH, Ohadi A (2017) Application of energies of optimal frequency bands for fault diagnosis based on modified distance function. J Mech Sci Technol 31(6):2701-2709

17. Medina R, Macancela JC, Lucero $P$, Cabrera D, Cerrada M, Sánchez RV, Vásquez RE (2019) Vibration signal analysis using symbolic dynamics for gearbox fault diagnosis. Int J Adv Manuf Technol 104:2195-2214

18. Hou L, Bergmann NW (2012) Novel industrial wireless sensor networks for machine condition monitoring and fault diagnosis. IEEE Trans Instrum Meas 61:2787-2798

19. Zhang ZJ, Chen CJ (2008) Tool condition monitoring in an end-milling operation based on the vibration signal collected through a microcontroller-based data acquisition system. Int J Adv Manuf Technol 39:118-128

20. Betta G, Liguori C, Paolillo A, Pietrosanto A (2002) A DSP-based FFT-analyzer for the fault diagnosis of rotating machine based on vibration analysis. IEEE Trans Instrum Meas 6:1316-1322

21. Camacho PYS, Ocampo JBR, Correa JCJ, Villalobos DJ (2015) FPGA-based reconfigurable system for tool condition monitoring in high-speed machining process. Measurement 64:81-88

22. Son J, Kang D, Boo D, Ko K (2018) An experimental study on the fault diagnosis of wind turbines through a condition monitoring system. J Mech Sci Technol 32(12):5573-5582

23. Goldman S (1999) Vibration spectrum analysis. Industrial Press, NY (USA)

24. SPM Instrument, Evaluated Vibration Analysis Method, http:// www.spminstrument.com/Measuring-techniques/Vibrationmonitoring/Vibration measurement-and-analysis, Accessed 18 July 2020.

25. D. Eberly, Information About Ellipses, https://www.geome trictools.com/Documentation/InformationAboutEllipses.pdf, Accessed 22 July 2020.

26. V. Spruyt, How to draw a covariance error ellipse?, https://www. visiondummy.com/2014/04/draw-error-ellipse-representingcovariance-matrix, Accessed 09 July 2020.

Publisher's Note Springer Nature remains neutral with regard to jurisdictional claims in published maps and institutional affiliations. 